\title{
慢性吗啡依赖树鼠模型的建立
}

\author{
孙永梅 ${ }^{1,2}$, 杨建珍 ${ }^{1}$, 孙华英 ${ }^{1}$, 马原野 ${ }^{1, *}$, 王建红 ${ }^{1, *}$ \\ (1. 中国科学院昆明动物研究所, 云南 昆明 650223; 2. 中国科学院研究生院, 北京 100049)
}

\begin{abstract}
摘要: 吗啡是一种有效的镇痛药, 但易使动物产生耐受性和成瘾性。在该实验中，中缅树鼠 (Tupaia belangeri chinensis)连续 $7 \mathrm{~d}$ ，每天接受三次肌肉注射递增剂量(5、10、15、20 mg/kg 体重)吗啡后对吗啡产生耐受和依赖; 吗 啡注射完成后, 腹腔注射纳洛酮(1.25 mg/kg 体重)催瘾, 可诱导其条件性位置厌恶(conditioned place aversion, CPA) 及相应吗啡戒断症状的出现。该结果提示树鼠慢性吗啡依赖模型的建立可用于研究吗啡依赖和耐受的生物学机制, 以及减轻戒断症状药物的笁选。
\end{abstract}

关键词：树獡；吗啡；慢性依赖；条件性位置厌恶；戒断症状

中图分类号: Q959.832; Q95-33; Q189 文献标志码: A

文章编号：0254-5853-(2012)01-0014-05

\section{Establishment of tree shrew chronic morphine dependent model}

\author{
SUN Yong-Mei ${ }^{1,2}$, YANG Jian-Zhen ${ }^{1}$, SUN Hua-Ying ${ }^{1}$, MA Yuan-Ye ${ }^{1, *}$, WANG Jian-Hong ${ }^{1, *}$ \\ (1. Kunming Institute of Zoology, the Chinese Academy of Sciences, Kunming, 650223, China; \\ 2. Graduate School of the Chinese Academy of Sciences, Beijing 100049, China)
}

\begin{abstract}
The clinical use of morphine to reduce pain is limited because of its drug tolerance, dependence and addiction. In the present study, the tree shrews (Tupaia belangeri chinensis) developed morphine tolerance and chronic morphine dependence by morphine injections with increasing doses $(5,10,15,20 \mathrm{mg} / \mathrm{kg}$ body weight for 7 days). Meanwhile, the naloxone $(1.25 \mathrm{mg} / \mathrm{kg}$ body weight) -induced conditioned place aversion (CPA) and the withdrawal symptom were also found. The tree shrew model of chronic morphine dependence can be used to investigate the withdrawal symptoms and to select potential withdrawal symptoms reducing drugs in the future.
\end{abstract}

Key words: Tree shrew; Morphine; Chronic dependence; Conditioned place aversion; Withdrawal symptom

吗啡作为常见的阿片类镇痛药物之一，被广泛 用于临床治疗各种急、慢性疼痛(Ree et al, 1999; Waldhoer et al, 2004; Martell et al, 2007), 但因可能 导致的耐受性(tolerance)、依赖性(dependence), 及 成瘾性，其应用受到限制(Ree et al, 1999; Martell et al，2007)。现代成瘾生物学的一个基本问题是：为 什么诸如吗啡、海洛因等的阿片类药物极易导致耐 受、依赖和成瘾(Waldhoer et al, 2004), 而药物依赖 又会导致药物滥用, 从而引发诸如社会暴力、艾滋 病、肝炎传染等一系列社会问题(Leshner, 1997)。通 过建立动物模型来研究药物依赖的生物学机制是 回答该问题的有效方法。
药物依赖指持续应用药物后, 个体生理状态发 生改变, 而停止药物应用后, 个体将产生戒断症状 (Ree et al, 1999)。判断动物对某种药物是否产生依 赖, 常通过停药后观察戒断症状, 或者注射该药物 的受体拮抗剂催滤等方法。如: 吗啡依赖的大、小 鼠在注射吗啡 4 $8 \mathrm{~h}$ 后, 腹腔注射阿片受体拮抗剂 纳洛酮会产生明显的戒断症状(Schulteis et al, 2004)。药物戒断后的条件性位置厌恶(conditioned place aversion, CPA)指由戒断所致的厌恶动机/情绪 与特定的环境/信号搭配后可建立条件性反射, 当 条件化后的对象再次暴露于相同或相似的环境/信 号时, 个体所表现出的戻恶/回避行为(Li et al, 2006,

收稿日期: 2011-12-19; 接受日期: 2012-01-05

基金项目: “973” 项目((2011CB707800); 中国科学院知识创新工程重要方向项目(KSCX2-EW-R-11)

*通信作者(Corresponding authors), E-mail: yuanma0716@vip.sina.com; wangjh@mail.kiz.ac.cn

第一作者简介：孙永梅，硕士研究生 
$\mathrm{Xu}$ et al, 2004)。因此, CPA 是一个判断动物是否产 生药物依赖及戒断症状的灵敏方法(Azar et al, 2003)。

树鼠属于攀鼠目(Scandentia)树鼠科(Tupaiinae), 虽然其系统分类地位仍有争议, 但它具备一些近似 原始灵长类的特征, 说明它与现代灵长类亲缘关系 很近(Peng et al, 1991; Sargis, 2004)。因此，树鼠的慢 性吗啡依赖模型较大、小鼠模型能够更好地模拟人 类药物依赖及戒断症状。

以树鼠为模型进行的药物研究数据非常有限。 Wiens et al (2008)发现由于马来西亚笔尾树鼠 (Ptilocercus lowii) 的特殊代谢系统, 使其在大量饮 用含高浓度酒精的花蜜后并不表现醉酒的状态，且 无宿醉行为特征; 而酒精易成瘾的大鼠品系对吗啡 也易成瘾(John et al, 1967)。那么, 同属树鼠科的中 缅树鼠是否也保留了该特殊代谢系统, 而该系统对 成瘾药物吗啡是否也有作用, 本文拟通过持续性吗 啡注射观察树鼠对吗啡产生的依赖及成瘾状况，从 而探讨树鼠吗啡依赖/成瘾模型的可行性及其在吗 啡、酒精成瘾研究方面的应用前景。

树鼠体型小、繁殖快、易饲养, 属昼行性动物, 活动高峰为 6:00-9:30 和 15:00-18:30; 视觉发达, 能清楚区别红、黄、绿和灰色，且红色对其具有最 大的刺激阈值(Peng et al, 1991)。

本实验结合树鼠的活动及视觉特征, 采用 $\mathrm{CPA}$ 装置, 通过纳洛酮诱导位置厌恶和戒断症状, 以探 讨其对吗啡的慢性依赖。

\section{1 材料和方法}

\section{1 实验动物}

24 只成年健康雄性中缅树鼠(Tupaia belangeri chinensis), 体重 110 146 g, 购于中国科学院昆明动 物研究所动物实验中心。动物单笼饲养在 $12 \mathrm{~h}$ 明暗 交替(光照时间 07:00-19:00)的动物房中，室温 $(23 \pm 1){ }^{\circ} \mathrm{C}$, 自由饮食。所有树鼣在开始实验前, 至少在动物房适应一周, 在此期间每天接受抚摸一 次。其中 2 只在 CPA 箱中穿梭次数 $<2$ 被剔除, 4 只注 射吗啡后死亡, 所以进行 CPA 数据分析的树鼣共 18 只。

\section{2 实验药品}

盐酸吗啡: 针剂, $10 \mathrm{mg} / \mathrm{mL}$, 沈阳第一制药厂, 批号 070802; 盐酸纳洛酮: 粉剂, Sigma-Aldrich, 批 号 2066110 , 注射当天用 $0.9 \%$ 生理盐水配至 1.25 $\mathrm{mg} / \mathrm{mL}$ 。

\section{3 实验装置}

$\mathrm{CPA}$ 箱：箱体为有机玻璃顶盖的木质箱，两个 可以活动的隔板将其分为三个箱体。两侧大箱体 $(33 \mathrm{~cm} \times 33 \mathrm{~cm} \times 33 \mathrm{~cm}$ ), 即条件化匹配箱壁和底板 分别为黑色和红色; 中间小箱体 $(40 \mathrm{~cm} \times 17 \mathrm{~cm} \times$ $33 \mathrm{~cm}$ ), 即选择箱壁和底板黑色和红色各半。箱体 上方 $1.5 \mathrm{~m}$ 高的天花板上悬挂记录动物行为的摄像 头, 监控数据传入置于另一房间的电脑中, 以便进 行离线分析。

戒断症状记录装置: 粉色铁丝笼 $(39 \mathrm{~cm} \times 27 \mathrm{~cm}$ $\times 29 \mathrm{~cm})$ 一只, 作为树鼠戒断场地; 磁带式摄像机 一部，用于记录树鼠戒断症状。

\section{4 行为测试和记录}

1.4.1 纳洛酩诱导慢性吗啡依赖树鼠的 CPA CPA 行为训练分为 3 个阶段：条件化前期、条件化期和 测试期。训练时间根据树鼠活动规律安排。实验动 物分为 3 组: 慢性吗啡注射 + 纳洛酮催瘾组 (morphine+naloxone, $\mathrm{MN}$ 组)、慢性吗啡注射+生理 盐水组 (morphine+saline, MS 组)和慢性生理盐水注 射+纳洛酮催瘾组( saline+naloxone, SN 组)。其中 MS 组和 SN 组为对照组。

条件化前期(第 1 天～第 5 天，D1～D5)：每天 8:00、14:00 和 20:00, MN 组和 MS 组分别肌肉注射 吗啡或者 SN 组肌肉注射等体积生理盐水, 剂量见 表 1。注射完毕后动物立即回到饲养笼。每天 16:00 将动物放入除去隔板的 $\mathrm{CPA}$ 箱体中, 使其自由活动 15 min。第 4 天和第 5 天的数据作为 CPA 分数基准 值。剔除进入任何一个条件化匹配箱的次数 $<2$ 的动 物，条件化期(第 6 天, D6): 该天动物的给药方式和 时间同条件化前期。但 3 组动物于 16:00 腹腔注射 $0.9 \%$ 生理盐水 $(1 \mathrm{~mL} / \mathrm{kg}$ 体重)后被立即限制在非偏 爱箱 $30 \mathrm{~min} ; 18: 00, \mathrm{MN}$ 组与 $\mathrm{SN}$ 组腹腔注射纳洛酮 $(1.25 \mathrm{mg} / \mathrm{kg}$ 体重); 而 $\mathrm{MS}$ 腹腔注射生理盐水后, 动 物被立即限制在偏爱箱 $30 \mathrm{~min}$ 。

测试期(第 7 天, D7): 该天动物的给药方式和时 间同条件化前期。16:00, CPA 装置的隔板被移除, 动 物在 $\mathrm{CPA}$ 箱体中自由活动 $15 \mathrm{~min}$, 录像记录其行为。 1.4.2 戒断症状的记录 戒断症状记录(第 8 天和 第 9 天, D8 和 D9): D8, 8:00 MN 组和 MS 组树鼠 肌肉注射吗啡 $(25 \mathrm{mg} / \mathrm{kg}$ 体重)并立即放回饲养笼中, $4 \mathrm{~h}$ 后(12:00), 两组动物腹腔注射生理盐水 $(1 \mathrm{~mL} / \mathrm{kg}$ 体重)后放入粉色铁丝笼中记录其行为 $15 \mathrm{~min}$, 然 后, $12: 15$ 腹腔注射纳洛酠(1.2 $5 \mathrm{mg} / \mathrm{kg}$ )后再记录其 
行为 $15 \mathrm{~min} 。 \mathrm{D} 9$ 对 $\mathrm{SN}$ 组树鼠分别记录腹腔注射生 理盐水和纳洛酮后的行为, 记录时间同 $\mathrm{D} 8$, 与 $\mathrm{D} 8$
不同之处是 8:00, SN 组树鼠肌肉注射生理盐水。 $\mathrm{CPA}$ 行为训练及戒断症状的记录安排详见表 1 。

表 1 实验日程和处理因素

Tab. 1 Experiment design and the schedule

\begin{tabular}{|c|c|c|c|c|c|}
\hline $\begin{array}{l}\text { 日期 } \\
\text { Date }\end{array}$ & $\begin{array}{l}\mathrm{MN} \text { 组注射 } \\
\text { Injection of } \\
\mathrm{MN} \text { group }\end{array}$ & $\begin{array}{l}\text { MS 组注射 } \\
\text { Injection of } \\
\text { MS group }\end{array}$ & $\begin{array}{l}\text { SN 组注射 } \\
\text { Injection of } \\
\text { SN group }\end{array}$ & $\begin{array}{c}\text { CPA 训练和戒断时间安排 } \\
\text { The schedule of CPA training and withdrawal }\end{array}$ & $\begin{array}{c}\text { 每日吗啡注射剂量 }(\mathrm{mg} / \mathrm{kg} \text { 体重 } \times \text { 次数 }) \\
\text { The morphine injection dose per } \\
\text { day }(\mathrm{mg} / \mathrm{kg} \text { body weight } \times \text { times })\end{array}$ \\
\hline D1 & M & M & $\mathrm{S}$ & \multirow{5}{*}{$\begin{array}{l}\text { 每日 16:00 开始条件化前训练 } \\
\text { The pre-conditioning training begins at 16:00 }\end{array}$} & $5 \times 3$ \\
\hline D2 & M & M & $\mathrm{S}$ & & $5 \times 3$ \\
\hline D3 & M & M & S & & $10 \times 3$ \\
\hline D4 & M & M & $\mathrm{S}$ & & $15 \times 3$ \\
\hline D5 & M & M & S & & $20 \times 3$ \\
\hline \multirow[t]{2}{*}{ D6 } & M & M & $\mathrm{S}$ & 16:00 开始，限制在非偏爱侧 $30 \mathrm{~min}$ & $20 \times 3$ \\
\hline & $\mathrm{S}$ & $\mathrm{S}$ & $S$ & $\begin{array}{l}\text { The tree shrews were confined in the non-preferring } \\
\text { box for } 30 \text { min from 16:00 } \\
18 ; 00 \text { 开始, 限制在偏爱侧 } 30 \text { min } \\
\text { The tree shrews were confined in the preferring box } \\
\text { for } 30 \text { min from 18:00 }\end{array}$ & \\
\hline D7 & M & M & $\mathrm{S}$ & $\begin{array}{c}16: 00 \mathrm{CPA} \text { 测试 } \\
\text { CPA tests began at 16:00 }\end{array}$ & $25 \times 3$ \\
\hline D8 & $\begin{array}{l}\mathrm{M} \\
+\mathrm{S}, \mathrm{N}\end{array}$ & $\begin{array}{c}\mathrm{M} \\
+\mathrm{S}, \mathrm{N}\end{array}$ & S & $\begin{array}{c}\text { 12:15 MN 组、MS 组注射纳洛酮观察戒断症状 } \\
\text { The recording of withdrawal symptoms of MN group } \\
\text { and MS group after naloxone injection from 12:15 }\end{array}$ & 25 \\
\hline D9 & & & $\begin{array}{c}\mathrm{S} \\
+\mathrm{S}, \mathrm{N}\end{array}$ & $\begin{array}{c}12: 15 \mathrm{SN} \text { 组注射纳洛酮观察症状 } \\
\text { The recording of withdrawal symptoms of SN group } \\
\text { after naloxone injection from 12:15 }\end{array}$ & \\
\hline
\end{tabular}

M: 吗啡(Morphine); N: 纳洛酮(Naloxone); S: 生理盐水(Saline)。

1.4.3 体重监测 行为测试期间, 每天 7:00-8:00 称量所有树鼠体重。

\section{5 数据处理}

$\mathrm{CPA}$ 数据处理: 统计动物停留在红色和黑色箱 体的时间(秒), 并依据以下公式转化为 $\mathrm{CPA}$ 分数 $\mathrm{R}$ 值。 $\mathrm{R}$ (红箱 CPA 分数 $)=$ 红箱内停留时间/(红箱内停 留时间+黑箱内停留时间)。如果 $\mathrm{R}>0.50$, 表示该动 物偏爱红色箱体, 则红箱为条件化期 18:00 的伴纳 洛酮箱, 黑箱为非偏爱箱; 若 $\mathrm{R}<0.50$, 则黑色箱体 为伴纳洛酮箱, 红色箱为非偏爱箱。

体重: $\mathrm{D} 1 \sim \mathrm{D} 5$ 的数据用于分析和比较, $\mathrm{D} 1$ 的 体重为基准值, 以 Mean $\pm S D$ 表示。

所有统计分析均用 SPSS 16.0 for Windows 进 行。CPA 分数以 Mean $\pm S E$ 表示,组内采用配对 $t$-检 验, 组间采用独立样本 $t$-检验。 $P \leqslant 0.05$ 表示在统 计上有显著性差异, $P \leqslant 0.01$ 表示有极显著差异。

\section{2 结 果}

\section{1 三组树鼠 CPA 评定结果}

$\mathrm{MN}$ 组经纳洛酮催瘾后, 对伴纳洛酮箱明显厌 恶 $(t=2.903, P=0.034)$; 虽然统计上无显著性, MS 组 $(t=3.18, P=0.13)$ 停留在偏爱箱的时间在条件化后增
多; 同样剂量的纳洛酩并没有导致 SN 组厌恶伴纳 洛酮箱 $(t=-2.038, P=0.134)$ (图 1)。以上数据说明, $\mathrm{MN}$ 组树舄产生庈恶并不是因为该剂量纳洛酮具有 使动物产生厌恶的作用，而是由于纳洛酮诱导了吗 啡依赖树䳔产生戒断症状，进而诱发了对伴纳洛酮 侧箱体的厌恶。

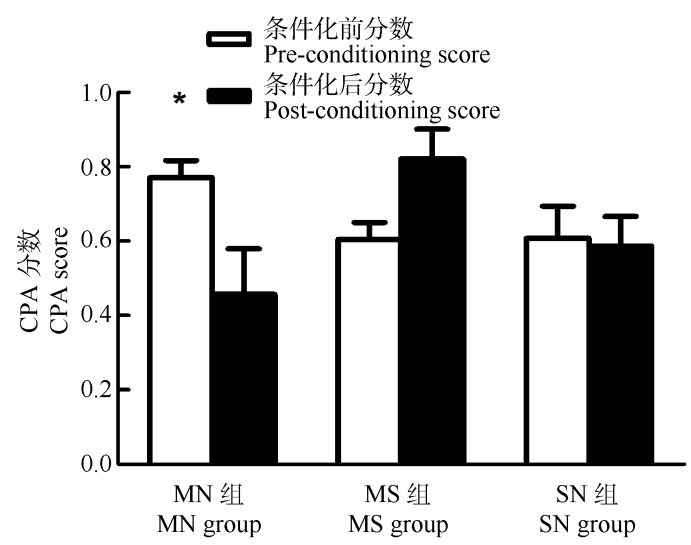

图 1 三组树鼠 CPA 分数

Fig. 1 The CPA score of three tree shrew groups * $P<0.05, \mathrm{MN}$ 组条件化前、后分数比较具显著性差异 ( $\mathrm{MN}$ 组: $n=6$; MS 组: $n=4$; SN 组: $n=8)$ 。

* $P<0.05$, MN group CPA score showed significant difference between pre-conditioning and post-conditioning session (MN group: $n=6$; MS group: $n=4$; SN group: $n=8$ ). 


\section{2 戒断症状分析与比较}

通过 $M S$ 组和 $M N$ 组自身对照以及与 $\mathrm{SN}$ 组的 组间对照, 我们观察到以下戒断症状, 并与大鼠的 戒断症状进行了比较 (表 2)。

表 2 树鼠和大鼠戒断症状比较

Tab. 2 Withdrawal symptoms comparison between rats and tree shrews

\begin{tabular}{|c|c|}
\hline & 大鼠 \\
\hline $\begin{array}{c}\text { Similarities } \\
\text { 相同点 }\end{array}$ & $\begin{array}{l}\text { 跳跃 Jumping、襍阴 Cunnilingus、扭体 Writhing、理毛 } \\
\text { Grooming、摇头 Shaking head、湿狗样抖 Wet dog shaking、 } \\
\text { 洗脸 Washing face、爪震颤 Paw trembling、甩尾 Drifting }\end{array}$ \\
\hline $\begin{array}{l}\text { Differences } \\
\text { 不同点 }\end{array}$ & $\begin{array}{lc}\text { 叫声 Yelling: 吹呱呱, 呱呱 Twee } & \text { 无(n/a) } \\
\text { 前、后爪拍地 Paw pattering } & \text { 无(n/a) } \\
\text { 倒立 Head-standing } & \text { 无(n/a) } \\
\text { 头、尾贴地走 Head grazing, tail 无(n/a) } \\
\text { grazing } & \text { 腹泻 Diarrhea } \\
\text { 无(n/a) } & \text { 吞咽 Swallowing } \\
\text { 无(n/a) } & \text { 磨牙 Grinding } \\
\text { 无(n/a) } & \end{array}$ \\
\hline
\end{tabular}

\section{3 吗啡注射后树鼠体重变化}

与基准值相比，吗啡组树鼠在注射吗啡 $1 \mathrm{~d}$ 后 体重急剧下降 $(t=8.698, P<0.001)$, 随后趋于平稳, 表明树鼠对吗啡产生了耐受(图 2)。生理盐水组树鼠 体重变化不明显。同时, 我们观察到在吗啡注射期 间，树鼠的饮食量与注射前、后相比均明显减少。

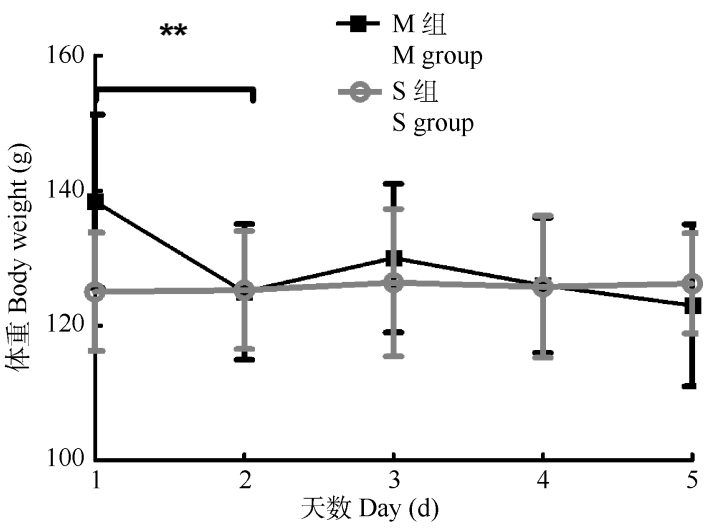

图 2 条件化前期吗啡组( $\mathrm{M}$ 组)和生理盐水组 $(\mathrm{S}$ 组) 树鼠体重变化

Fig. 2 Body weight changes in $\mathrm{M}$ group and $\mathrm{S}$ group during pre-conditioning session

** $P<0.01$, 第 1 次吗啡注射前、后体重比较, 有显著性差异。( $\mathrm{M}$ 组: $n=10, \mathrm{~S}$ 组: $n=8)$ 。

** $P<0.01$, body weight showed significant difference between before and after the first morphine injection. (M group: $n=10, \mathrm{~S}$ group: $n=8$ ).

2.4 条件化前期吗啡注射树鼠对红色箱体的偏爱 程度

条件化前期第 4、5 天，与生理盐水组相比，吗
啡注射树鼣表现出对红色箱体的极显著偏爱 $(t=3.454, P=0.003)$ (图 3)。

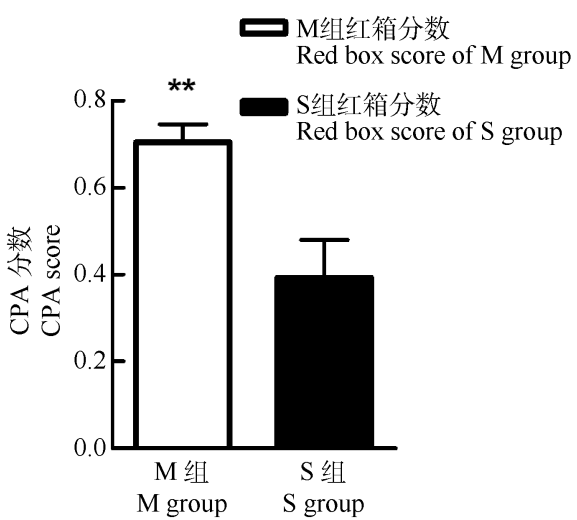

图 3 条件化前期吗啡组( $M$ 组)和生理盐水组 $(\mathrm{S}$ 组) 动物的红色箱体停留分数

Fig. 3 Red box scores of M group and S group in pre-conditioning session

${ }^{* *} P<0.01$ (M 组: $n=10, \mathrm{~S}$ 组: $n=8$ )。

$* * P<0.01$ (M group: $n=10, \mathrm{~S}$ group: $n=8$ ).

\section{3 讨 论}

本实验发现，每天三次注射递增剂量吗啡，树 鼠能够对吗啡产生一定的耐受和依赖，通过纳络酮 催瘾，可观察到戒断症状，并促使树鼠形成 CPA。

正性和负性强化机制在药物依赖中具重要作 用(Tiffany et al, 2004)。药物的奖赏作用是正性强化 因素，而药物戒断所产生的戒断症状及厌恶情绪是 负性强化因素。在本实验中，正性强化因素是吗啡 注射，而负性强化因素是纳络酮催瘫产生的戒断症 状，以及与戒断症状相关的环境因素。实验中我们 发现: $M N$ 组树鼠与 $M S$ 组、 $\mathrm{SN}$ 组比较, 明显表明 出对伴纳洛酮箱体的回避。该结果与啮齿类动物相 关报道一致(Marc et al, 2003; Karyn et al, 2010)。这 说明树鼠对吗啡产生依赖后用阿片受体拮抗剂纳 洛酮催瘾，会由于戒断症状而对催瘾相关的环境线 索产生回避行为, 且该模型可作为研究药物成瘾及 戒断的有效方法之一。

人类阿片类毒品戒断症状包括呕吐、打呵欠、 流眼泪、腹泻、腹痛、蚂蚁爬骨的异样感觉、睡眠 障碍及抑郁等。树鼠的戒断症状与人类有一定的相 似之处。比如：树鼠戒断过程中，叫声和倒立反映 其烦躁不安情绪，与人类的心理戒断症状相似; 前、后爪拍地，与人类的不宁腿综合征相似; 头、 尾贴地走，与人类的 “静坐不能” 症状相似; 但因 
为动物种属不同, 树鼠的某些戒断症状无法和人类 相比较, 而未观察到树鼠腹泻现象, 原因可能是由 于树鼣在面对新异环境或刺激时，强烈的应激反应 可能使它们对吗啡的耐受增高，同时肠胃功能也能 够迅速恢复。在本实验中, 树鼠的体重在注射吗啡 初始迅速下降, 之后便趋于平稳, 这从另一方面也 说明, 树鼠消化道功能的适应能力很强, 因而没有 产生腹泻症状。该推测需要通过进一步实验验证。 另外, 催瘾症状与纳洛酮的剂量有关, 还需要相关 实验探讨不同剂量的纳洛酮是否能诱导树鼣腹泻 等症状。

$\mathrm{CPA}$ 和戒断症状都是判断动物是否产生药物 依赖的指标。药物成瘾的核心特征是强迫性药物使 用(Leshner, 1997)。因此, 药物依赖模型可以揭示成 滤是怎样从随机地、有控制地用药到强迫性用药。

以往的研究发现，啮齿类动物体重的减轻可以 作为吗啡依赖的指标。本实验发现，树鼠在吗啡给 药一天后也出现了体重的显著降低, 说明体重减轻 也可作为其吗啡依赖的指标之一。但是，在之后的 给药期间树鼠体重波动不明显，提示其对吗啡产生 耐受，而耐受是限制吗啡临床使用的因素之一，因 此，中缅树鼠吗啡依赖模型也可用于研究吗啡耐受 机制。

\section{参考文献:}

Azar MR, Jones BC, Schulteis G. 2003. Conditioned place aversion is a highly sensitive index of acute opioid dependence and withdrawal[J]. Psychopharmacology, 170(1): 42-50.

Deroche-Gamonet V, Belin D, Piazza PV. 2004. Evidence for addiction-like behavior in the rat[J]. Science, 305(5686): 1014-1017.

John R. Nichols JR, Hsiao S. 1967. Addiction liability of albino rats: breeding for quantitative differences in morphine drinking[J]. Science, 157(3788): 561-563

Leshner AI. 1997. Addiction is a brain disease, and it matters[J]. Science, 278(5335): 45-47.

Li Y, Hao W, Wang XY, Deng H.Q. 2006. Developing the conditioned place aversion rat model produced by naloxone-precipitated withdrawal from chronic morphine dependence[J]. Chn J Clin Psychol, 14(3): 275-278. [李毅, 郝伟, 王绪轶, 邓慧琼, 2006. 慢性吗啡成瘾大鼠纳洛酮催 瘾戒断后 CPA 模型的建立 [J]. 中国临床心理学杂志, 14(3): 275-277.]

Martell BA, O'Connor PG, Kerns RD, Becker WC, Morales KH, Kosten TR, Fiellin DA. 2007. Systematic review: opioid treatment for chronic back pain: prevalence, efficacy, and association with addiction[J]. Ann Intern Med, 146(2): 116-127.

Myers KM, Carlezon WA Jr. 2010. D-cycloserine facilitates extinction of naloxone-induced conditioned place aversion in morphine-dependent rats[J]. Biol Psychiatry, 67(1): 85-87
中缅树鼠可以对吗啡产生依赖并出现戒断症 状的一个重要因素可能是给药方式。我们在前期的 预实验中发现，两种恒定剂量 $(10 \mathrm{mg} / \mathrm{kg}$ 体重 和 15 $\mathrm{mg} / \mathrm{kg}$ 体重), 每天腹腔注射一次, 连续 $8 \mathrm{~d}$ 的给药 方式不能建立 CPA。首次注射剂量太高 $(15 \mathrm{mg} / \mathrm{kg}$ 体重)，树鼠死亡率高达 75\%，而剂量太低，树獡句又 易产生耐受。因而，我们选用每日 3 次递增剂量给 药方式，使吗啡药效作用时间延长，并能避免因药 量大和药效快导致的动物急性死亡。

由于黑色所给予的安全感，树鼠天生偏爱黑色， 但吗啡注射后的树舄对红色具显著偏爱，这可能是 因为吗啡解除了中枢抑制系统的作用，从而使中枢 神经系统兴奋而导致树鼠对红色箱的偏爱。

药物伴随(包括戒断时)的环境因素是导致复吸 的重要因素之一(Deroche-Gamonet et al, 2004)。因 此，纳洛酮诱导的慢性吗啡 CPA 模型不仅可以用于 探讨戒断症状在成瘧过程中的作用机理，也可用来 探讨相关环境线索是怎样影响复吸的。另外，该模 型也可以用来篮选减轻吗啡戒断症状的药物。本研 究通过 CPA、戒断症状观察和体重监测等方法，初 步建立了中缅树鼠的吗啡依赖模型，且由于树鼣进 化地位及脑结构较啮齿类动物均更接近灵长类，该 模型具有广大的应用前景。

Peng YZ, Ye ZZ, Zou RJ, Wang YX, Tian BP, Ma YY, Shi LM. 1991. Biology of Chinese Tree Shrews[M]. Kunming, China: Yunnan Science and Technology Press. [彭燕章, 叶智彰, 邹如金,王应祥, 田保平, 马原野, 施立明. 1991. 树鼠生物学. 昆明: 云南科技出版社.]

Sargis EJ. 2004. New views on tree shrews: the role of Tupaiids in primate supraordinal relationships[J]. Evol Anthropol, 13(2): 56-66.

Schulteis G, Morse AC, Liu J. 2004. Conditioning processes contribute to severity of naloxone-precipitated withdrawal from acute opioid dependence[J]. Psychopharmacology, 175(4): 463-472.

van Ree JM, Gerrits MAFM, Vanderschuren LJMJ. 1999. Opioids, reward and addiction: an encounter of biology, psychology, and medicine $[\mathrm{J}]$. Pharmacol Rev, 51(2): 341-396.

Waldhoer M, Bartlett SE, Whistler JL. 2004. Opioid receptors[J]. Annu Rev Biochem, 73: 953-990.

Wiens F, Zitzmann A, Lachance MA, Yegles M, Pragst F, Wurst FM, holst D, Guan SL, Spanagel R. 2008. Chronic intake of fermented floral nectar by wild tree shrews[J]. Proc Natl Acad Sci USA, 105(30): 10426-10431.

Xu W, Luo XJ, Tan BP, Zheng XG, Yang XY, Sui N. 2004. Effect of naloxone on conditioned place aversion in morphine-treated rats[J]. Chn J Pain Med, 10(2): 105-109. [须卫, 罗小景, 谭北平, 邓希耕, 杨晓燕, 隋南. 2004. 纳洛酮诱发吗啡成瘾大鼠 CPA 模型的建立[J]. 中国疼痛医学杂志, 10(2): 105-109.] 